\title{
LOGARITHMIC CONCAVITY, UNITARITY AND SELFADJOINTNESS
}

\author{
JAN STOCHEL \\ Institute of Mathematics, Jagiellonian University, 30-059 Kraków, Poland \\ E-mail: stochel@im.uj.edu.pl
}

Dedicated to Professor W. Żelazko on the occasion of his seventieth birthday

\begin{abstract}
Isometric automorphisms of normed linear spaces are characterized by suitable concavity properties of powers of operators. Bounded selfadjoint operators in Hilbert spaces are described by parallel concavity properties of the exponential group. Unbounded infinitesimal generators of $\mathcal{C}_{0}$-groups of Hilbert space operators having concavity properties are characterized as well.
\end{abstract}

1. Introduction. From now on, $\mathbb{Z}$ stands for the set of all integers and $\mathbb{R}$ for the field of all real numbers. Recall that a sequence $\left\{a_{n}\right\}_{n=0}^{\infty} \subseteq \mathbb{R} \cup\{-\infty\}$ is concave if

$$
\frac{1}{2}\left(a_{n}+a_{n+2}\right) \leqslant a_{n+1}, \quad n \geqslant 0 .
$$

A sequence $\left\{a_{n}\right\}_{n=0}^{\infty}$ of nonnegative real numbers is logarithmically concave if

$$
\sqrt{a_{n} \cdot a_{n+2}} \leqslant a_{n+1}, \quad n \geqslant 0,
$$

or equivalently if the sequence $\left\{\log a_{n}\right\}_{n=0}^{\infty}$ is concave (with the obvious convention $\log 0=-\infty)$. Each concave sequence $\left\{a_{n}\right\}_{n=0}^{\infty}$ of nonnegative real numbers is automatically logarithmically concave because

$$
\sqrt{a_{n} \cdot a_{n+2}} \leqslant \frac{1}{2}\left(a_{n}+a_{n+2}\right) \leqslant a_{n+1}, \quad n \geqslant 0 .
$$

The case of bilateral sequences can be handled in much the same way. Given $\left\{a_{n}\right\}_{n \in \mathbb{Z}} \subseteq$ $[0, \infty)$, we write $\sum_{n=0}^{\infty} a_{ \pm n}=\infty$ in case $\sum_{n=0}^{\infty} a_{n}=\infty$ and $\sum_{n=0}^{\infty} a_{-n}=\infty$. Similar convention applies to the case of the $\operatorname{limit}_{n \rightarrow \infty} \lim _{ \pm n}$.

2000 Mathematics Subject Classification: Primary 47B15; Secondary 47B20.

Key words and phrases: isometry, unitary operator, selfadjoint operator, concave function (sequence), logarithmically concave function (sequence).

This work was supported by the KBN grant \# 2 P03A 037024.

The paper is in final form and no version of it will be published elsewhere. 
Given a (nonzero) normed linear space $\mathcal{X}$, we denote by $\boldsymbol{B}(\mathcal{X})$ the algebra of all bounded linear operators on $\mathcal{X}$. We write $I$ for the identity operator on $\mathcal{X}$. If $\mathcal{X}$ is a Banach space and $A \in \boldsymbol{B}(\mathcal{X})$, then $r(A)$ stands for the spectral radius of $A$. By the Naimark-Beurling-Gelfand theorem (cf. [12, Section II] for the case of real Banach spaces), we have

$$
r(A)=\lim _{n \rightarrow \infty}\left\|A^{n}\right\|^{1 / n} .
$$

Paranormal operators were introduced in $[10,9]$ as generalizations of hyponormal ones. An operator $A \in \boldsymbol{B}(\mathcal{X})$ is called paranormal if

$$
\|A x\|^{2} \leqslant\left\|A^{2} x\right\| \cdot\|x\|, \quad x \in \mathcal{X} .
$$

It turns out that $A$ is paranormal if and only if the sequence $\left\{\left\|A^{n} x\right\|\right\}_{n=0}^{\infty}$ is logarithmically convex for all $x \in \mathcal{X}$. Such operators, even though $A^{-1} \in \boldsymbol{B}(\mathcal{X})$, are far from being isometric. The study of seminormal composition operators on $L^{2}$-spaces with affine symbols led the authors of [7] to a question how to describe operators $A \in \boldsymbol{B}(\mathcal{X})$ which satisfy the inequality

$$
\left\|A^{2} x\right\| \cdot\|x\| \leqslant\|A x\|^{2}, \quad x \in \mathcal{X}
$$

which is, in a sense, opposite to (1.3). The condition (1.4) is equivalent to requiring that the sequence $\left\{\left\|A^{n} x\right\|\right\}_{n=0}^{\infty}$ is logarithmically concave for all $x \in \mathcal{X}$. It was proved in [7, Lemma 4.1] that if $\mathcal{X}$ is a finite dimensional Hilbert space and $A \in \boldsymbol{B}(\mathcal{X})$ is a bijection satisfying (1.4), then $A$ is a scalar multiple of a unitary operator. We show that this statement still remains valid for infinite dimensional (real or complex) normed linear spaces $\mathcal{X}$ provided "a unitary operator" is replaced by "an isometric automorphism of $\mathcal{X}$ " (cf. Theorem 3.3). In other words, the logarithmic concavity of sequences of the form $\left\{\left\|A^{n} x\right\|\right\}_{n=0}^{\infty}, x \in \mathcal{X}$, completely characterizes scalar multiples of isometric automorphisms of $\mathcal{X}$ amongst all members of the algebra $\boldsymbol{B}(\mathcal{X})$. This in turn leads to a characterization of isometric automorphisms of $\mathcal{X}$ (cf. Theorem 3.4).

A new class of operators $A \in \boldsymbol{B}(\mathcal{X})$ satisfying the following inequality

$$
\left\|A^{2} x\right\|^{2}+\|x\|^{2} \leqslant 2\|A x\|^{2}, \quad x \in \mathcal{X}
$$

has been introduced and studied by Richter in [14] on the occasion of investigation of invariant subspaces of the Dirichlet shift. Operators $A$ satisfying (1.5) have also appeared implicitly in [17] on the occasion of investigation of composition operators on $L^{2}$-spaces with nonsingular matrix symbols (apply $[17,(2.9)]$ to $\phi(z)=\mathrm{e}^{z}, z \in \mathbb{C}$ ). The inequality (1.5) is easily seen to be equivalent to requiring that the sequence $\left\{\left\|A^{n} x\right\|^{2}\right\}_{n=0}^{\infty}$ is concave for all $x \in \mathcal{X}$. Such operators are called 2-hyperexpansive (cf. [2]). As noticed in [11, Remark 6.9], 2-hyperexpansive operators always satisfy (1.4). A very particular case of Proposition 3.5 (which in turn follows from a much more general result, Proposition 2.4) shows that a linear bijection $A$ of a normed linear space $\mathcal{X}$ onto itself is an isometric automorphism of $\mathcal{X}$ if and only if $A$ satisfies (1.5) (cf. [16, Remark 3] for the case of bounded Hilbert space operators); in other words, the concavity of sequences of the form $\left\{\left\|A^{n} x\right\|^{2}\right\}_{n=0}^{\infty}, x \in \mathcal{X}$, completely characterizes isometric automorphisms of $\mathcal{X}$ amongst linear bijections of $\mathcal{X}$ onto $\mathcal{X}$. We have decided to include two independent proofs of Proposition 2.4 one of which is based on an idea found in [14]; another one fits into 
our general scheme presented in Section 2. In fact, this result has nothing to do with topological and linear structures of $\mathcal{X}$.

Theorem 3.3 enables us to characterize purely imaginary (scalar) translations of bounded selfadjoint operators: a Hilbert space operator $T \in \boldsymbol{B}(\mathcal{H})$ is a purely imaginary translation of a selfadjoint operator if and only if the function $\mathbb{R} \ni t \mapsto \log \left\|\mathrm{e}^{\mathrm{i} t T} h\right\|$ is concave for all $h \in \mathcal{H}$ (cf. Theorem 4.1). This leads to a characterization of bounded selfadjoint operators (cf. Theorem 4.3). Finally, we deduce that for every real $p \neq 0$, the concavity of functions of the form $\mathbb{R} \ni t \mapsto\left\|\mathrm{e}^{\mathrm{i} t T} h\right\|^{p}, h \in \mathcal{H} \backslash\{0\}$, completely characterizes selfadjoint operators $T \in \boldsymbol{B}(\mathcal{H})$ (cf. Theorem 4.4). The content of Section 4 is closely related to Theorem 2 of [8] (see also [3, 4]) which states that a Hilbert space operator $T \in \boldsymbol{B}(\mathcal{H})$ is normal if and only if the functions $\mathbb{R} \ni t \mapsto \log \left\|\mathrm{e}^{\mathrm{i} t T} h\right\|$ and $\mathbb{R} \ni t \mapsto \log \left\|\mathrm{e}^{\mathrm{i} t T^{*}} h\right\|$ are convex for all $h \in \mathcal{H} \backslash\{0\}$. Section 5 extends the results of Section 4 to the case of unbounded infinitesimal generators of $\mathcal{C}_{0}$-groups of Hilbert space operators.

2. Preparatory facts. This section deals with mappings on sets which are not assumed to be normed linear spaces (what is more no linear structure is required). In fact, the results of this section can be appropriately adapted to the context of real sequences which are either logarithmically concave (resp. logarithmically convex) or concave (resp. convex). The details are left to the reader.

Lemma 2.1. Let $\phi$ be a positive function on a set $\Omega$ and let $A: \Omega \rightarrow \Omega$ be a mapping such that

$$
\phi\left(A^{2}(x)\right) \cdot \phi(x) \leqslant \phi(A(x))^{2}, \quad x \in \Omega .
$$

Then the following conditions are valid:

(i) for every $x \in \Omega$, the sequence $\left\{\frac{\phi\left(A^{n+1}(x)\right)}{\phi\left(A^{n}(x)\right)}\right\}_{n=0}^{\infty}$ is monotonically decreasing, the limits $\lim _{n \rightarrow \infty} \frac{\phi\left(A^{n+1}(x)\right)}{\phi\left(A^{n}(x)\right)}$ and $\lim _{n \rightarrow \infty} \sqrt[n]{\phi\left(A^{n}(x)\right)}$ exist and

$$
\lim _{n \rightarrow \infty} \frac{\phi\left(A^{n+1}(x)\right)}{\phi\left(A^{n}(x)\right)}=\lim _{n \rightarrow \infty} \sqrt[n]{\phi\left(A^{n}(x)\right)}<\infty,
$$

(ii) the condition (2.1) holds with $A^{k}$ in place of $A$ for all integers $k \geqslant 0$,

(iii) if $x \in \Omega$ is such that $\lim _{n \rightarrow \infty} \sqrt[n]{\phi\left(A^{n}(x)\right)} \geqslant 1$, then $\phi(A(x)) \geqslant \phi(x)$.

Moreover, if $A$ is a bijection, then

(iv) the condition (2.1) holds with $A^{k}$ in place of $A$ for all integers $k$,

$(\mathrm{v})$ if $x \in \Omega$ is such that ${ }^{1} \lim _{n \rightarrow \infty} \sqrt[n]{\phi\left(A^{n}(x)\right) \cdot \phi\left(A^{-n}(x)\right)} \geqslant 1$, then ${ }^{2}$

$$
\phi\left(A^{n}(x)\right)=\left(\frac{\phi(A(x))}{\phi(x)}\right)^{n} \cdot \phi(x), \quad n \in \mathbb{Z},
$$

(vi) if $\phi\left(A^{2}(x)\right) \cdot \phi(x)=\phi(A(x))^{2}$ for all $x \in \Omega$, then (2.3) holds for all $x \in \Omega$ and $\lim _{n \rightarrow \infty} \sqrt[n]{\phi\left(A^{n}(x)\right) \cdot \phi\left(A^{-n}(x)\right)}=1$ for all $x \in \Omega$

${ }^{1}$ By (i) and (iv) the $\operatorname{limit}_{\lim } \rightarrow \infty \sqrt[n]{\phi\left(A^{n}(x)\right) \cdot \phi\left(A^{-n}(x)\right)}$ always exists.

${ }^{2}$ Substituting $n=2$ into $(2.3)$, we get $\phi\left(A^{2}(x)\right) \cdot \phi(x)=\phi(A(x))^{2}$. 
(vii) if $x \in \Omega$ is such that $\lim _{n \rightarrow \infty} \sqrt[n]{\phi\left(A^{ \pm n}(x)\right)} \geqslant 1$, then $\phi(A(x))=\phi(x)$.

Proof. (i) Replacing $x$ by $A^{n}(x)$ in (2.1) and dividing both sides of the resulting inequality by $\phi\left(A^{n}(x)\right) \cdot \phi\left(A^{n+1}(x)\right)$, we obtain

$$
0<\frac{\phi\left(A^{n+2}(x)\right)}{\phi\left(A^{n+1}(x)\right)} \leqslant \frac{\phi\left(A^{n+1}(x)\right)}{\phi\left(A^{n}(x)\right)}, \quad n \geqslant 0,
$$

which guarantees that the $\operatorname{limit}_{n \rightarrow \infty} \frac{\phi\left(A^{n+1}(x)\right)}{\phi\left(A^{n}(x)\right)}$ exists and is finite. One can show that this in turn implies ${ }^{3}$ (2.2).

(ii) If $k \geqslant 2$, then by (i) we have

$$
\begin{aligned}
\frac{\phi\left(A^{2 k}(x)\right)}{\phi\left(A^{k}(x)\right)} & =\frac{\phi\left(A^{2 k}(x)\right)}{\phi\left(A^{2 k-1}(x)\right)} \cdots \frac{\phi\left(A^{k+1}(x)\right)}{\phi\left(A^{k}(x)\right)} \\
& \leqslant \frac{\phi\left(A^{k}(x)\right)}{\phi\left(A^{k-1}(x)\right)} \cdots \frac{\phi(A(x))}{\phi(x)}=\frac{\phi\left(A^{k}(x)\right)}{\phi(x)}, \quad x \in \Omega,
\end{aligned}
$$

which implies that (2.1) holds with $A^{k}$ in place of $A$.

(iv) Replacing $x$ by $A^{-2}(x)$ in $(2.1)$, we see that (2.1) holds with $A^{-1}$ in place of $A$. If we combine this with (ii), we get (iv).

(iii) By (i) we have

$$
\frac{\phi(A(x))}{\phi(x)} \geqslant \lim _{n \rightarrow \infty} \frac{\phi\left(A^{n+1}(x)\right)}{\phi\left(A^{n}(x)\right)}=\lim _{n \rightarrow \infty} \sqrt[n]{\phi\left(A^{n}(x)\right)} \geqslant 1 .
$$

(v) Given $0<\theta \leqslant 1$ and $y \in \Omega$, we denote by $\Xi_{\theta}^{y}$ the set of all bijections $C: \Omega \rightarrow \Omega$ satisfying the following two inequalities:

$$
\begin{aligned}
& \phi(C(x)) \cdot \phi\left(C^{-1}(x)\right) \leqslant \phi(x)^{2}, \quad x \in \Omega, \\
& \phi(C(y)) \cdot \phi\left(C^{-1}(y)\right) \leqslant \theta \cdot \phi(y)^{2} .
\end{aligned}
$$

We show that

$$
C \in \Xi_{\theta}^{y} \Longrightarrow C^{2} \in \Xi_{\theta^{2}}^{y} .
$$

Indeed, replacing $x$ by $C(x)$ (resp. $x$ by $\left.C^{-1}(x)\right)$ in (2.4) we get

$$
\begin{gathered}
\phi\left(C^{2}(x)\right) \cdot \phi(x) \leqslant \phi(C(x))^{2}, \\
\phi(x) \cdot \phi\left(C^{-2}(x)\right) \leqslant \phi\left(C^{-1}(x)\right)^{2} .
\end{gathered}
$$

Multiplying the above two inequalities and applying (2.4) we obtain

$$
\phi\left(C^{2}(x)\right) \cdot \phi\left(C^{-2}(x)\right) \cdot \phi(x)^{2} \leqslant \phi(C(x))^{2} \cdot \phi\left(C^{-1}(x)\right)^{2} \leqslant \phi(x)^{4}, \quad x \in \Omega .
$$

Since $\phi(x)>0$ for $x \in \Omega$, we conclude that (2.4) is valid if we replace $C$ by $C^{2}$. It follows from (2.7) and (2.5) that

$$
\phi\left(C^{2}(y)\right) \cdot \phi\left(C^{-2}(y)\right) \cdot \phi(y)^{2} \leqslant \phi(C(y))^{2} \cdot \phi\left(C^{-1}(y)\right)^{2} \leqslant \theta^{2} \cdot \phi(y)^{4},
$$

which gives us (2.5) with $C^{2}$ in place of $C$ and $\theta^{2}$ in place of $\theta$. This proves (2.6).

\footnotetext{
${ }^{3}$ Pass to logarithms, then apply the fact that the sequence of arithmetic means of a given convergent sequence is convergent to the same limit, and finally go back via the exponent function.
} 
Replacing $x$ by $A^{-1}(x)$ in (2.1) we see that (2.4) holds for $C=A$. Take $x \in \Omega$ such that $\lim _{n \rightarrow \infty} \sqrt[n]{\phi\left(A^{n}(x)\right) \cdot \phi\left(A^{-n}(x)\right)} \geqslant 1$. We claim that

$$
\phi(A(x)) \cdot \phi\left(A^{-1}(x)\right)=\phi(x)^{2} .
$$

Indeed, otherwise there exists $0<\theta<1$ such that $A \in \Xi_{\theta}^{x}$. It follows from (2.6) that $A^{2^{n}} \in \Xi_{\theta^{2}}^{x}$ for very $n \geqslant 1$, i.e.

$$
\phi\left(A^{2^{n}}(x)\right) \cdot \phi\left(A^{-2^{n}}(x)\right) \leqslant \theta^{2^{n}} \cdot \phi(x)^{2} .
$$

This and $\phi(x)>0$ imply

$$
1 \leqslant \lim _{n \rightarrow \infty} \sqrt[2^{n}]{\phi\left(A^{2^{n}}(x)\right) \cdot \phi\left(A^{-2^{n}}(x)\right)} \leqslant \theta \cdot \lim _{n \rightarrow \infty} \sqrt[2^{n}]{\phi(x)^{2}}=\theta<1,
$$

which is impossible. This proves our claim.

Using (i) and (iv), one can verify that for every integer $k$, the point $x_{k}:=A^{k}(x)$ has the same property as $x$, i.e. $\lim _{n \rightarrow \infty} \sqrt[n]{\phi\left(A^{n}\left(x_{k}\right)\right) \cdot \phi\left(A^{-n}\left(x_{k}\right)\right)} \geqslant 1$. By what has been proved in the previous paragraph, we have

$$
\phi\left(A\left(A^{k}(x)\right)\right) \cdot \phi\left(A^{-1}\left(A^{k}(x)\right)\right)=\phi\left(A^{k}(x)\right)^{2}, \quad k \in \mathbb{Z} .
$$

Substituting $k=n+1$ into (2.9), we deduce that

$$
\frac{\phi\left(A^{n+2}(x)\right)}{\phi\left(A^{n+1}(x)\right)}=\frac{\phi\left(A^{n+1}(x)\right)}{\phi\left(A^{n}(x)\right)}, \quad n \in \mathbb{Z},
$$

which leads to

$$
\phi\left(A^{n}(x)\right)=\frac{\phi\left(A^{n}(x)\right)}{\phi\left(A^{n-1}(x)\right)} \cdots \frac{\phi(A(x))}{\phi(x)} \cdot \phi(x)=\left(\frac{\phi(A(x))}{\phi(x)}\right)^{n} \cdot \phi(x), \quad n \geqslant 1 .
$$

Applying (2.10) to $A^{-1}$ in place of $A$ (which is possible due to (iv)) and exploiting (2.8), we conclude that

$$
\phi\left(A^{-n}(x)\right)=\left(\frac{\phi\left(A^{-1}(x)\right)}{\phi(x)}\right)^{n} \cdot \phi(x)=\left(\frac{\phi(A(x))}{\phi(x)}\right)^{-n} \cdot \phi(x), \quad n \geqslant 1 .
$$

Following the above argument, one can establish (vi).

The condition (vii) is a consequence of (v) (it can be also deduced from (iii) and (iv)). This completes the proof.

Replacing $\phi$ by $1 / \phi$ we get the "dual" version of Lemma 2.1 (the "dual" version of (vi) is not stated explicitly because it coincides with the original one). Notice that (2.1) corresponds to (1.4) while (2.11) to (1.3).

Lemma 2.2. Let $\phi: \Omega \rightarrow(0, \infty)$ and $A: \Omega \rightarrow \Omega$ be such that

$$
\phi\left(A^{2}(x)\right) \cdot \phi(x) \geqslant \phi(A(x))^{2}, \quad x \in \Omega .
$$

Then the following conditions are valid:

$\left(\mathrm{i}^{*}\right)$ for every $x \in \Omega$, the sequence $\left\{\frac{\phi\left(A^{n+1}(x)\right)}{\phi\left(A^{n}(x)\right)}\right\}_{n=0}^{\infty}$ is monotonically increasing, the limits $\lim _{n \rightarrow \infty} \frac{\phi\left(A^{n+1}(x)\right)}{\phi\left(A^{n}(x)\right)}$ and $\lim _{n \rightarrow \infty} \sqrt[n]{\phi\left(A^{n}(x)\right)}$ exist in $(0, \infty]$ and

$$
\lim _{n \rightarrow \infty} \frac{\phi\left(A^{n+1}(x)\right)}{\phi\left(A^{n}(x)\right)}=\lim _{n \rightarrow \infty} \sqrt[n]{\phi\left(A^{n}(x)\right)},
$$

(ii*) the condition (2.11) holds with $A^{k}$ in place of $A$ for all integers $k \geqslant 0$, 
(iii*) if $x \in \Omega$ is such that $\lim _{n \rightarrow \infty} \sqrt[n]{\phi\left(A^{n}(x)\right)} \leqslant 1$, then $\phi(A(x)) \leqslant \phi(x)$. Moreover, if $A$ is a bijection, then

(iv*) the condition (2.11) holds with $A^{k}$ in place of $A$ for all integers $k$,

$\left(\mathrm{v}^{*}\right)$ if $x \in \Omega$ is such that $\lim _{n \rightarrow \infty} \sqrt[n]{\phi\left(A^{n}(x)\right) \cdot \phi\left(A^{-n}(x)\right)} \leqslant 1$, then

$$
\phi\left(A^{n}(x)\right)=\left(\frac{\phi(A(x))}{\phi(x)}\right)^{n} \cdot \phi(x), \quad n \in \mathbb{Z},
$$

(vii*) if $x \in \Omega$ is such that $\lim _{n \rightarrow \infty} \sqrt[n]{\phi\left(A^{ \pm n}(x)\right)} \leqslant 1$, then $\phi(A(x))=\phi(x)$.

REMARK 2.3. The condition (v) of Lemma 2.1 need not imply $\phi(A(x))=\phi(x)$ nor even $\phi(A(x)) \geqslant \phi(x)$ (cf. Theorem 3.3). The conditions (iii) and (vii) of Lemma 2.1 yield the following:

(iii' $^{\prime}$ if $x \in \Omega$ is such that $\sum_{n=0}^{\infty} \phi\left(A^{n}(x)\right)=\infty$, then $\phi(A(x)) \geqslant \phi(x)$,

$\left(\right.$ vii $\left.^{\prime}\right)$ if $x \in \Omega$ is such that $\sum_{n=0}^{\infty} \phi\left(A^{ \pm n}(x)\right)=\infty$, then $\phi(A(x))=\phi(x)$.

Replacing $\phi$ by $1 / \phi$ in (2.1) we can formulate the "dual" versions of (iii') and (vii').

Proposition 2.4. Let $\phi$ be a nonnegative function on a set $\Omega$ and let $A: \Omega \rightarrow \Omega$ be a bijection. Then the following two conditions are equivalent:

$1^{\circ} \phi(A(x))=\phi(x)$ for every $x \in \Omega$,

$2^{\circ} \phi(A(x))+\phi\left(A^{-1}(x)\right) \leqslant 2 \phi(x)$ for every $x \in \Omega$.

Proof I of Proposition 2.4. The implication $1^{\circ} \Rightarrow 2^{\circ}$ is obvious.

$2^{\circ} \Rightarrow 1^{\circ}$ Define the function $\psi: \Omega \rightarrow[0, \infty)$ by $\psi(x)=\mathrm{e}^{\phi(x)}$ for $x \in \Omega$. Applying part (vii) of Lemma 2.1 to $\psi$ in place of $\phi$ completes the proof.

Proof II of Proposition 2.4. The implication $2^{\circ} \Rightarrow 1^{\circ}$ of Proposition 2.4 can be also proved independently of Lemma 2.1 as follows ${ }^{4}$. Replacing $x$ by $A(x)$ in $2^{\circ}$, we get $\phi\left(A^{2}(x)\right)+$ $\phi(x) \leqslant 2 \phi(A(x))$ for all $x \in \Omega$. This implies

$$
\phi\left(A^{2}(x)\right)-\phi(A(x)) \leqslant \phi(A(x))-\phi(x), \quad x \in \Omega .
$$

Replacing $x$ by $A^{k}(x)$ leads to

$$
\phi\left(A^{k+2}(x)\right)-\phi\left(A^{k+1}(x)\right) \leqslant \phi\left(A^{k+1}(x)\right)-\phi\left(A^{k}(x)\right), \quad x \in \Omega, k \geqslant 0 .
$$

Hence we have

$$
\begin{aligned}
0 \leqslant \phi\left(A^{n}(x)\right) & =\sum_{j=1}^{n}\left(\phi\left(A^{j}(x)\right)-\phi\left(A^{j-1}(x)\right)\right)+\phi(x) \\
& \leqslant n(\phi(A(x))-\phi(x))+\phi(x) \\
& =n \phi(A(x))+(1-n) \phi(x), \quad x \in \Omega, n \geqslant 1
\end{aligned}
$$

which implies

$$
\phi(A(x)) \geqslant \frac{n-1}{n} \cdot \phi(x), \quad x \in \Omega, n \geqslant 1
$$

\footnotetext{
${ }^{4}$ Our proof is based upon an idea found in the proof of [14, Lemma 1].
} 
Letting $n \rightarrow \infty$ yields

$$
\phi(A(x)) \geqslant \phi(x), \quad x \in \Omega .
$$

Since $2^{\circ}$ holds with $A^{-1}$ in place of $A$, the above reasoning enables us to write $\phi\left(A^{-1}(x)\right)$ $\geqslant \phi(x)$ for $x \in \Omega$. Replacing $x$ by $A(x)$ gives us $\phi(x) \geqslant \phi(A(x))$ for $x \in \Omega$, which when combined with (2.12) completes the proof.

One could expect Lemma 2.1 to be deduced from Proposition 2.4 applied to $\log \phi$; however this is not the case because $\log \phi$ may not be positive.

3. Isometric automorphisms. A bounded linear operator $A$ on a Banach space $\mathcal{X}$ is called normaloid if $r(A)=\|A\|$ (cf. [5]). Before proving the main result of this section, we will show that a nonzero normaloid $A$ which satisfies (1.4) is a scalar multiple of an isometry.

Lemma 3.1. If $\mathcal{X}$ is a (real or complex) Banach space and $A \in \boldsymbol{B}(\mathcal{X})$ is a nonzero normaloid which satisfies (1.4), then $\|A\|^{-1} A$ is an isometry.

Proof. Without loss of generality we can assume that $\|A\|=1$. Set

$$
\Omega=\left\{x \in \mathcal{X}: A^{n} x \neq 0 \text { for all } n \geqslant 0\right\} .
$$

It is easily seen that $A(\Omega) \subseteq \Omega$ and

$$
\lim _{n \rightarrow \infty} \sqrt[n]{\left\|A^{n} x\right\|}=0, \quad x \in \mathcal{X} \backslash \Omega
$$

Denote by $\mathcal{X}_{0}$ the set of all $x \in \mathcal{X}$ such that $\limsup _{n \rightarrow \infty} \sqrt[n]{\left\|A^{n} x\right\|}=r(A)=1$. It follows from (3.1) that $\mathcal{X}_{0} \subseteq \Omega$. Applying parts (i) and (iii) of Lemma 2.1 to the function $\phi(x)=\|x\|, x \in \Omega$, and to the mapping $\left.A\right|_{\Omega}$, we get

$$
\|A x\| \geqslant\|x\|, \quad x \in \mathcal{X}_{0} .
$$

By [6, Theorem, p. 181] (see also [18] and [12, Corollary 1.2]) and [12, Corollary 2.4], the set $\mathcal{X}_{0}$ is dense in $\mathcal{X}$. This and (3.2) imply that $\|A x\| \geqslant\|x\|$ for all $x \in \mathcal{X}$. Since $\|A\|=1$, we conclude that $A$ is an isometry.

Remark 3.2. Notice that every nonzero operator $A \in \boldsymbol{B}(\mathcal{X})$ whose square vanishes satisfies (1.4), though $0=r(A)<\|A\|$ and $A$ is not a scalar multiple of an isometry ${ }^{5}$. On the other hand, if $\mathcal{X}$ is a normed linear space and $A: \mathcal{X} \rightarrow \mathcal{X}$ is a linear mapping satisfying (1.4) and $A^{2} \neq 0$, then $A$ is an injection. Indeed, taking $x, y \in \mathcal{X}$ such that $A^{2} x \neq 0$ and $A y=0$, we infer from (1.4) that

$$
\left\|A^{2} x\right\| \cdot\|x+t y\| \leqslant\|A x\|^{2}, \quad t \in \mathbb{R}
$$

which implies $y=0$. Consequently, every linear surjection $A: \mathcal{X} \rightarrow \mathcal{X}$ satisfying (1.4) is a bijection and every operator $A \in \boldsymbol{B}(\mathcal{X})$ with dense range, which satisfies (1.4) is an injection.

${ }^{5}$ One can construct a closed unbounded linear operator $A$ in a Hilbert space $\mathcal{H}$ such that $A \mathcal{D}(A) \subseteq \mathcal{D}(A)$ and $A^{2} x=0$ for all $x \in \mathcal{D}(A)$, where $\mathcal{D}(A)$ is the domain of $A$ (cf. [13]); such an $A$ satisfies (1.4) with $\mathcal{X}=\mathcal{D}(A)$. 
TheOREM 3.3. If $\mathcal{X}$ is a nonzero (real or complex) normed linear space and $A: \mathcal{X} \rightarrow \mathcal{X}$ is a bijection such that either $A \in \boldsymbol{B}(\mathcal{X})$ or $A^{-1} \in \boldsymbol{B}(\mathcal{X})$, then the following conditions are equivalent:

(i) there exists a (unique) pair $(\lambda, U)$ composed of a positive real number $\lambda$ and an isometric automorphism $U \in \boldsymbol{B}(\mathcal{X})$ such that $A=\lambda \cdot U$,

(ii) A satisfies (1.4),

(iii) the sequence $\left\{\log \left\|A^{n} x\right\|\right\}_{n=0}^{\infty}$ is concave for every $x \in \mathcal{X} \backslash\{0\}$,

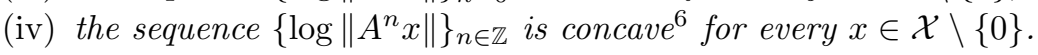

Proof. The implications (i) $\Rightarrow($ iv), (iv) $\Rightarrow$ (iii) and (iii) $\Rightarrow$ (ii) are easily seen to be true.

(ii) $\Rightarrow$ (i) Notice first that if $A \in \boldsymbol{B}(\mathcal{X})$ is a bijection and $\mathcal{X} \neq\{0\}$, then

$$
\inf _{x \neq 0} \frac{\left\|A^{-1} x\right\|}{\|x\|}=\frac{1}{\sup _{x \neq 0} \frac{\|x\|}{\left\|A^{-1} x\right\|}}=\frac{1}{\sup _{x \neq 0} \frac{\|A x\|}{\|x\|}}=\frac{1}{\|A\|} .
$$

We claim that

$$
\text { if a bijection } A \in \boldsymbol{B}(\mathcal{X}) \text { satisfies (1.4), then }\left\|A^{2^{n}}\right\|=\|A\|^{2^{n}} \text { for all } n \geqslant 0 \text {. }
$$

Indeed, replacing $x$ by $A^{-2} x$ in (1.4), then dividing both sides of so obtained inequality by $\|x\|^{2}$, and finally taking infimum over all $x \in \mathcal{X} \backslash\{0\}$ we get

$$
\inf _{x \neq 0} \frac{\left\|A^{-2} x\right\|}{\|x\|} \leqslant\left(\inf _{x \neq 0} \frac{\left\|A^{-1} x\right\|}{\|x\|}\right)^{2} .
$$

This and (3.3) (applied to $A$ and $A^{2}$ ) lead to $\|A\|^{2} \leqslant\left\|A^{2}\right\| \leqslant\|A\|^{2}$. Thus we have $\left\|A^{2}\right\|=\|A\|^{2}$. The latter equality and the fact that (1.4) holds with $A^{2}$ in place of $A$ (cf. part (ii) of Lemma 2.1), enable us to proceed by induction. In consequence, we get (3.4).

Suppose now that $A \in \boldsymbol{B}(\mathcal{X})$ is a bijection satisfying (1.4). Let $\overline{\mathcal{X}}$ be a completion of $\mathcal{X}$ and let $\bar{A} \in \boldsymbol{B}(\overline{\mathcal{X}})$ be a unique (continuous and linear) extension of $A$. Then (1.4) holds with $\overline{\mathcal{X}}$ and $\bar{A}$ in place of $\mathcal{X}$ and $A$, respectively. By (3.4) and (1.2), we have $r(\bar{A})=\|\bar{A}\|>0$. Applying Lemma 3.1 completes the proof of the case $A \in \boldsymbol{B}(\mathcal{X})$; the other one $A^{-1} \in \boldsymbol{B}(\mathcal{X})$ can be inferred from the first. The uniqueness of the decomposition $A=\lambda \cdot U$ is plain.

We are now in a position to formulate a characterization of isometric automorphisms of normed linear spaces.

THEOREM 3.4. If $\mathcal{X}$ is a nonzero (real or complex) normed linear space and $A: \mathcal{X} \rightarrow \mathcal{X}$ is a bijection such that either $A \in \boldsymbol{B}(\mathcal{X})$ or $A^{-1} \in \boldsymbol{B}(\mathcal{X})$, then the following conditions are equivalent:

(i) $A$ is an isometric automorphism of $\mathcal{X}$,

(ii) A satisfies (1.4) and there exists a unit vector $\xi \in \mathcal{X}$ such that $\|A \xi\|=1$,

(iii) the sequence $\left\{\log \left\|A^{n} x\right\|\right\}_{n=0}^{\infty}$ is concave for every $x \in \mathcal{X} \backslash\{0\}$ and there exists a unit vector $\xi \in \mathcal{X}$ such that $\|A \xi\|=1$,

\footnotetext{
${ }^{6}$ In fact, $\log \left\|A^{n} x\right\|$ is affine in variable $n$.
} 
(iv) the sequence $\left\{\log \left\|A^{n} x\right\|\right\}_{n \in \mathbb{Z}}$ is concave for every $x \in \mathcal{X} \backslash\{0\}$ and there exists a unit vector $\xi \in \mathcal{X}$ such that $\|A \xi\|=1$.

Proof. If (ii) holds, then by Theorem 3.3 there exists $\lambda>0$ and an isometric automorphism $U \in \boldsymbol{B}(\mathcal{X})$ such that $A=\lambda \cdot U$. Then

$$
1=\|A \xi\|=\lambda \cdot\|U \xi\|=\lambda \cdot\|\xi\|=\lambda
$$

which implies $A=U$.

The next characterization of isometric automorphisms of normed linear spaces is a direct consequence of Proposition 2.4 (with $\Omega=\mathcal{X} \backslash\{0\}$ and $\phi(x)=\|x\|^{p}$ ).

Proposition 3.5. If $\mathcal{X}$ is a nonzero (real or complex) normed linear space, $A: \mathcal{X} \rightarrow \mathcal{X}$ is a linear bijection and $p$ is a nonzero real number, then the following conditions are equivalent:

(i) $A$ is an isometric automorphism of $\mathcal{X}$,

(ii) A satisfies the following inequality:

$$
\|A x\|^{p}+\left\|A^{-1} x\right\|^{p} \leqslant 2\|x\|^{p}, \quad x \in \mathcal{X} \backslash\{0\},
$$

(iii) the sequence $\left\{\left\|A^{n} x\right\|^{p}\right\}_{n=0}^{\infty}$ is concave for every $x \in \mathcal{X} \backslash\{0\}$,

(iv) the sequence $\left\{\left\|A^{n} x\right\|^{p}\right\}_{n \in \mathbb{Z}}$ is concave for every $x \in \mathcal{X} \backslash\{0\}$.

Remark 3.6. If $\mathcal{X}$ is a Banach space, $A \in \boldsymbol{B}(\mathcal{X})$ has a dense range and

$$
\left\|A^{2} x\right\|^{p}+\|x\|^{p} \leqslant 2\|A x\|^{p}, \quad x \in \mathcal{X},
$$

for some $p>0$, then $\|A x\| \geqslant 2^{-1 / p}\|x\|$ for all $x \in \mathcal{X}$, which implies that $A$ is an injection with closed and dense range. Hence $A$ is a bijection and consequently by Proposition 3.5, $A$ is an isometric automorphism of $\mathcal{X}$ (cf. [16, Remark 3] for the case $p=2$ ).

REMARK 3.7. Let $\mathcal{X}$ be a nonzero pre-Hilbert space and let $A: \mathcal{X} \rightarrow \mathcal{X}$ be a linear bijection. Define the linear operator $T: \mathcal{X} \rightarrow \mathcal{X} \oplus \mathcal{X}$ via $T x=A x \oplus A^{-1} x$ for $x \in \mathcal{X}$. By Proposition 3.5 (with $p=2$ ), the operator $A$ is unitary if and only if $\|T\| \leqslant \sqrt{2}$; if this is the case, then $2^{-1 / 2} \cdot T$ is an isometry.

A version of Proposition 3.5 for $p>0$ and for a bijection $A \in \boldsymbol{B}(\mathcal{X})$ can be deduced from Theorem 3.3 (apply (1.1) to $a_{n}=\left\|A^{n} x\right\|^{p}$ ). This suggests a question: is Theorem 3.3 true without assuming the continuity of $A$ and $A^{-1}$ ? Below we give some partial answers to the question.

Section 2 enables us to find circumstances under which the inequality (1.4) (or the inequality (1.3)) turns into the equality.

Proposition 3.8. If $\mathcal{X}$ is a nonzero (real or complex) normed linear space and $A: \mathcal{X} \rightarrow$ $\mathcal{X}$ is a bijection such that $A(0)=0$, then the following conditions are equivalent:

(i) $\left\|A^{2} x\right\| \cdot\|x\|=\|A x\|^{2}$ for all $x \in \mathcal{X}$,

(ii) A satisfies (1.4) and $\lim _{n \rightarrow \infty} \sqrt[n]{\left\|A^{n} x\right\| \cdot\left\|A^{-n} x\right\|} \geqslant 1$ for all $x \in \mathcal{X} \backslash\{0\}$,

(iii) A satisfies (1.3) and $\lim _{n \rightarrow \infty} \sqrt[n]{\left\|A^{n} x\right\| \cdot\left\|A^{-n} x\right\|} \leqslant 1$ for all $x \in \mathcal{X} \backslash\{0\}$.

Proof. Apply Lemmata 2.1 and 2.2 to $\phi(x)=\|x\|, x \in \mathcal{X} \backslash\{0\}$. 
Notice that if $A \in \boldsymbol{B}(\mathcal{X})$ satisfies the condition (i) of Proposition 3.8, then

$$
0 \leqslant\left(\left\|A^{2} x\right\|-\|x\|\right)^{2}=\left\|A^{2} x\right\|^{2}-2\|A x\|^{2}+\|x\|^{2}, \quad x \in \mathcal{X},
$$

which means that $A$ is a 2-hypercontraction (cf. [1]).

Following Lemmata 2.1 and 2.2, and Remark 2.3, we can characterize isometric automorphisms of normed linear spaces in terms of (1.4) (or (1.3)) and some extra conditions.

Proposition 3.9. If $\mathcal{X}$ is a nonzero (real or complex) normed linear space and $A: \mathcal{X} \rightarrow$ $\mathcal{X}$ is a linear bijection, then the following conditions are equivalent:

(i) $A$ is an isometric automorphism of $\mathcal{X}$,

(ii) A satisfies (1.4) and $\lim _{n \rightarrow \infty} \sqrt[n]{\left\|A^{ \pm n} x\right\|} \geqslant 1$ for all $x \in \mathcal{X} \backslash\{0\}$,

(iii) $A$ satisfies (1.4) and $\sum_{n=0}^{\infty}\left\|A^{ \pm n} x\right\|=\infty$ for all $x \in \mathcal{X} \backslash\{0\}$,

(iv) A satisfies (1.3) and $\lim _{n \rightarrow \infty} \sqrt[n]{\left\|A^{ \pm n} x\right\|} \leqslant 1$ for all $x \in \mathcal{X} \backslash\{0\}$,

(v) $A$ satisfies (1.3) and $\sum_{n=0}^{\infty}\left\|A^{ \pm n} x\right\|^{-1}=\infty$ for all $x \in \mathcal{X} \backslash\{0\}$.

Notice that the condition (1.3) alone is not sufficient for an invertible element $A$ of the algebra $\boldsymbol{B}(\mathcal{X})$ to be a scalar multiple of an isometric automorphism of $\mathcal{X}$ (even though $\mathcal{X}$ is a Hilbert space).

One can deduce directly from (1.4) that the sequence $\left\{\log \left\|A^{n}\right\|\right\}_{n \in \mathbb{Z}}$ is convex (see the proof of Theorem 3.3), i.e.

$$
\log \left\|A^{n+1}\right\| \leqslant \frac{1}{2}\left(\log \left\|A^{n}\right\|+\log \left\|A^{n+2}\right\|\right), \quad n \in \mathbb{Z} .
$$

Actually, by Theorem $3.3, \log \left\|A^{n}\right\|$ is linear in variable $n$.

4. Selfadjoint operators - the bounded case. In this section, applying the results of Section 3, we characterize purely imaginary translations of bounded selfadjoint operators.

THEOREM 4.1. If $\mathcal{H}$ is a nonzero complex Hilbert space and $T \in \boldsymbol{B}(\mathcal{H})$, then the following conditions are equivalent:

(i) there exists $r \in \mathbb{R}$ such that the operator $T+\mathrm{i} r I$ is selfadjoint,

(ii) $\left\|e^{\mathrm{i} t T} h\right\| \cdot\left\|e^{-\mathrm{i} t T} h\right\| \leqslant\|h\|^{2}$ for all $h \in \mathcal{H}$ and for all $t \in \mathbb{R}$,

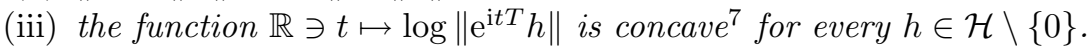

REMARK 4.2. A close inspection of the proof of Theorem 4.1 reveals that the conditions (i), (ii) and (iii) are still equivalent if we allow the real parameter $t$ to run through an interval (which is independent of $h$ ) with 0 in its centre. The same observation can be made in the context of Theorems 4.3 and 4.4 .

Notice also that if for every $h \in \mathcal{H} \backslash\{0\}$, there exists $\varepsilon_{h}>0$ such that the function $\mathbb{R} \ni t \mapsto \varphi_{h}(t):=\log \left\|\mathrm{e}^{\mathrm{i} t T} h\right\|$ is concave on the interval $\left(-\varepsilon_{h}, \varepsilon_{h}\right)$, then the condition (iii) of Theorem 4.1 holds. Indeed, since $\varphi_{g} \in \mathcal{C}^{\infty}(\mathbb{R})$ for every $g \in \mathcal{H} \backslash\{0\}$, and $\varphi_{\mathrm{e}^{\mathrm{i} s T} h}$ is concave on $\left(-\varepsilon_{\mathrm{e}^{\mathrm{i} s T h}}, \varepsilon_{\mathrm{e}^{\mathrm{i} s T} h}\right)$, we get $\varphi_{h}^{\prime \prime}(s)=\varphi_{\mathrm{e}^{\mathrm{i} s T h}}^{\prime \prime}(0) \leqslant 0$ for every $s \in \mathbb{R}$. Hence $\varphi_{h}$ is concave on the whole $\mathbb{R}$. Similar reasoning applies to the condition $3^{\circ}$ of Theorem 4.4 .

\footnotetext{
${ }^{7}$ In fact, the function $\mathbb{R} \ni t \mapsto \log \left\|\mathrm{e}^{\mathrm{i} t T} h\right\|$ is affine.
} 
Proof of Theorem 4.1. (i) $\Rightarrow$ (iii) By our assumption $T$ is of the form $T=S-\mathrm{i} r I$, where $S$ is a selfadjoint operator on $\mathcal{H}$. Since for every $t \in \mathbb{R}$, the operator $\mathrm{e}^{\mathrm{i} t S}$ is unitary, we see that

$$
\log \left\|\mathrm{e}^{\mathrm{i} t T} h\right\|=\log \left\|\mathrm{e}^{r t} \mathrm{e}^{\mathrm{i} t S} h\right\|=\log \left(\mathrm{e}^{r t} \cdot\|h\|\right)=r t+\log \|h\|, \quad h \in \mathcal{H} \backslash\{0\}, t \in \mathbb{R} .
$$

(iii) $\Rightarrow$ (ii) Write the definition of concavity of the function $t \mapsto \log \left\|\mathrm{e}^{\mathrm{i} t T} h\right\|$ at the points $-t, 0$ and $t$.

(ii) $\Rightarrow$ (i) Applying Theorem 3.3 to the operator $A=\mathrm{e}^{\mathrm{i} t T}$, we find for each $t \in \mathbb{R}$ a unique pair $(\lambda(t), U(t))$ composed of a positive real number $\lambda(t)$ and a unitary operator $U(t) \in \boldsymbol{B}(\mathcal{H})$ such that

$$
\mathrm{e}^{\mathrm{i} t T}=\lambda(t) \cdot U(t), \quad t \in \mathbb{R} .
$$

Set $\mu(t)=\lambda(t)^{2}$ for $t \in \mathbb{R}$. It follows from (4.1) that

$$
\mathrm{e}^{\mathrm{i} t T} \mathrm{e}^{-\mathrm{i} t T^{*}}=\mathrm{e}^{\mathrm{i} t T}\left(\mathrm{e}^{\mathrm{i} t T}\right)^{*}=\mu(t) U(t) U(t)^{*}=\mu(t) \cdot I, \quad t \in \mathbb{R} .
$$

This implies that the real valued function $\mu$ is differentiable on $\mathbb{R}$. Differentiating both sides of (4.2) leads to

$$
\mathrm{i} T \mathrm{e}^{\mathrm{i} t T} \mathrm{e}^{-\mathrm{i} t T^{*}}-\mathrm{ie}^{\mathrm{i} t T} T^{*} \mathrm{e}^{-\mathrm{i} t T^{*}}=\mu^{\prime}(t) \cdot I, \quad t \in \mathbb{R} .
$$

Substituting $t=0$ into (4.3), we obtain $\mathrm{i} T-\mathrm{i} T^{*}=\mu^{\prime}(0) \cdot I$, which means that the imaginary part of $T$ is equal to $-\frac{\mu^{\prime}(0)}{2} \cdot I$. Hence, we have $T=\operatorname{Re} T-\mathrm{i} \frac{\mu^{\prime}(0)}{2} \cdot I$, where $\operatorname{Re} T=\frac{1}{2}\left(T+T^{*}\right)$ and $\mu^{\prime}(0) \in \mathbb{R}$. This completes the proof.

The following two results characterize bounded selfadjoint operators by suitable concavity properties of the exponential group.

THEOREM 4.3. If $\mathcal{H}$ is a nonzero complex Hilbert space and $T \in \boldsymbol{B}(\mathcal{H})$, then the following conditions are equivalent:

(i) $T$ is selfadjoint,

(ii) $\left\|e^{\mathrm{i} t T} h\right\| \cdot\left\|e^{-\mathrm{i} t T} h\right\| \leqslant\|h\|^{2}$ for all $h \in \mathcal{H}$ and for all $t \in \mathbb{R}$, and there exist a unit vector $\xi \in \mathcal{H}$ and $\kappa \in \mathbb{R} \backslash\{0\}$ such that $\left\|\mathrm{e}^{\mathrm{i} \kappa T} \xi\right\|=1$,

(iii) the function $\mathbb{R} \ni t \mapsto \log \left\|\mathrm{e}^{\mathrm{i} t T} h\right\|$ is concave for all $h \in \mathcal{H} \backslash\{0\}$, and there exist a unit vector $\xi \in \mathcal{H}$ and $\kappa \in \mathbb{R} \backslash\{0\}$ such that $\left\|\mathrm{e}^{\mathrm{i} \kappa T} \xi\right\|=1$.

Proof. We only need to prove that (ii) implies (i). By Theorem 4.1, $T$ is of the form $T=$ $S-\mathrm{i} r I$, where $S \in \boldsymbol{B}(\mathcal{H})$ is selfadjoint and $r \in \mathbb{R}$. Hence we have $f(t):=\log \left\|\mathrm{e}^{\mathrm{i} t T} \xi\right\|=r t$ for all $t \in \mathbb{R}$. Since $f(\kappa)=0$ and $\kappa \neq 0$, we conclude that $r=0$, which completes the proof.

THEOREM 4.4. If $\mathcal{H}$ is a nonzero complex Hilbert space, $p$ is a nonzero real number and $T \in \boldsymbol{B}(\mathcal{H})$, then the following conditions are equivalent:

$1^{\circ} T$ is selfadjoint,

$2^{\circ}\left\|e^{\mathrm{i} t T} h\right\|^{p}+\left\|e^{-\mathrm{i} t T} h\right\|^{p} \leqslant 2\|h\|^{p}$ for all $h \in \mathcal{H} \backslash\{0\}$ and for all $t \in \mathbb{R}$,

$3^{\circ}$ the function $\mathbb{R} \ni t \mapsto\left\|\mathrm{e}^{\mathrm{i} t T} h\right\|^{p}$ is concave for every $h \in \mathcal{H} \backslash\{0\}$.

Proof. The implications $1^{\circ} \Rightarrow 3^{\circ}$ and $3^{\circ} \Rightarrow 2^{\circ}$ are easily seen to be true. 
$2^{\circ} \Rightarrow 1^{\circ}$ By Proposition 3.5 the operator $\mathrm{e}^{\mathrm{i} t T}$ is unitary for each $t \in \mathbb{R}$. Applying Stone's theorem (or repeating the proof of the implication (ii) $\Rightarrow$ (i) of Theorem 4.1 with $\lambda(t) \equiv 1$ ), we conclude that the operator $T$ is selfadjoint.

It is well-known that a Hilbert space operator $T \in \boldsymbol{B}(\mathcal{H})$ is selfadjoint if and only if $\left\|\mathrm{e}^{\mathrm{i} t T}\right\|=1$ for all $t \in \mathbb{R}$. The following is a variant of this characterization.

Corollary 4.5. If $\mathcal{H}$ is a nonzero complex Hilbert space, then an operator $T \in \boldsymbol{B}(\mathcal{H})$ is selfadjoint if and only if for every $h \in \mathcal{H}$, the function $t \mapsto\left\|\mathrm{e}^{\mathrm{i} t T} h\right\|$ is constant on $\mathbb{R}$ (equivalently: on some neighborhood of 0 ).

Proof I of Corollary 4.5. Apply Theorem 4.4.

Proof II of Corollary 4.5. We only need to prove the "if" part of the conclusion. Since the function $t \mapsto\left\|\mathrm{e}^{\mathrm{i} t T} h\right\|^{2}=\left\langle\mathrm{e}^{-\mathrm{i} t T^{*}} \mathrm{e}^{\mathrm{i} t T} h, h\right\rangle$ is constant, its derivative vanishes, i.e.

$$
\left\langle\left(-\mathrm{i} T^{*} \mathrm{e}^{-\mathrm{i} t T^{*}} \mathrm{e}^{\mathrm{i} t T}+\mathrm{ie}^{-\mathrm{i} t T^{*}} T \mathrm{e}^{\mathrm{i} t T}\right) h, h\right\rangle=0, \quad h \in \mathcal{H}, t \in \mathbb{R} .
$$

Substituting $t=0$, we get $\left\langle\left(\mathrm{i} T-\mathrm{i} T^{*}\right) h, h\right\rangle=0$ for all $h \in \mathcal{H}$, which is equivalent to $T^{*}=T$. This completes the proof.

5. Selfadjoint operators - the unbounded case. The results of Section 4 can be formulated for generators of $C_{0}$-groups of Hilbert space operators. We begin with a $\mathcal{C}_{0^{-}}$ group analogue of Theorems 4.1 and 4.3 .

TheOREM 5.1. Let $\mathcal{H}$ be a nonzero complex Hilbert space and let $T$ be an infinitesimal generator of a $\mathcal{C}_{0}$-group $\{G(t): t \in \mathbb{R}\} \subseteq \boldsymbol{B}(\mathcal{H})$. Then the following conditions are equivalent:

(i) there exists $r \in \mathbb{R}$ such that the operator $\mathrm{i}(T+r I)$ is selfadjoint,

(ii) $\|G(t) h\| \cdot\|G(-t) h\| \leqslant\|h\|^{2}$ for all $h \in \mathcal{H}$ and for all $t \in \mathbb{R}$,

(iii) the function $\mathbb{R} \ni t \mapsto \log \|G(t) h\|$ is concave for every $h \in \mathcal{H} \backslash\{0\}$.

The operator i $T$ is selfadjoint if and only if either of the conditions (ii) and (iii) holds and there exist a unit vector $\xi \in \mathcal{H}$ and $\kappa \in \mathbb{R} \backslash\{0\}$ such that $\|G(\kappa) \xi\|=1$.

Proof. (i) $\Rightarrow$ (iii) If $T=\mathrm{i} S+r I$, where $S$ is selfadjoint and $r \in \mathbb{R}$, then $G(t)=\mathrm{e}^{r t} \mathrm{e}^{\mathrm{i} t S}$ for all $t \in \mathbb{R}$. In consequence, $\log \|G(t) h\|=r t+\log \|h\|$ for $h \in \mathcal{H} \backslash\{0\}$.

(ii) $\Rightarrow$ (i) By Theorem 3.3, for every $t \in \mathbb{R}$ there exists a unique pair $(\lambda(t), U(t))$ composed of a positive real number $\lambda(t)$ and a unitary operator $U(t) \in \boldsymbol{B}(\mathcal{H})$ such that

$$
G(t)=\lambda(t) \cdot U(t), \quad t \in \mathbb{R} .
$$

Fix a unit vector $\xi$ in $\mathcal{H}$. According to (5.1) we have

$$
\lambda(t)=\|G(t) \xi\|, \quad t \in \mathbb{R} .
$$

Since $\{G(t): t \in \mathbb{R}\}$ is a $\mathcal{C}_{0}$-group, (5.2) guarantees the continuity of $\mathbb{R} \ni t \rightarrow \lambda(t)$. It follows from (5.1) and the unitarity of the operators $U(s), s \in \mathbb{R}$, that

$$
\begin{aligned}
\lambda(s+t) & =\|\lambda(s+t) U(s+t)\|=\|G(s+t)\|=\|G(s) G(t)\| \\
& =\|\lambda(s) \lambda(t) U(s) U(t)\|=\lambda(s) \lambda(t), \quad s, t \in \mathbb{R} .
\end{aligned}
$$


By the above and $\lambda(0)=1$, we conclude that $\lambda(t)=\mathrm{e}^{r t}$, where $r=\log \lambda(1) \in \mathbb{R}$. This combined with (5.1) implies that $\mathbb{R} \ni t \rightarrow U(t)=\mathrm{e}^{-r t} G(t)$ is a $\mathcal{C}_{0}$-group of unitary operators. Applying Stone's theorem (cf. [15, Section 137]) we find a selfadjoint operator $S$ in $\mathcal{H}$ such that $U(t)=\mathrm{e}^{\mathrm{i} t S}$ for all $t \in \mathbb{R}$. Hence $G(t)=\mathrm{e}^{r t} \mathrm{e}^{\mathrm{i} t S}$ for all $t \in \mathbb{R}$, which gives (i).

The rest of the proof follows very closely the proof of Theorem 4.3.

Applying Proposition 3.5 and Stone's theorem (cf. [15, Section 137]) we get a $\mathcal{C}_{0}$-group analogue of Theorem 4.4 .

THEOREM 5.2. If $\mathcal{H}$ is a nonzero complex Hilbert space, $T$ is an infinitesimal generator of a $\mathcal{C}_{0}$-group $\{G(t): t \in \mathbb{R}\} \subseteq \boldsymbol{B}(\mathcal{H})$ and $p$ is a nonzero real number, then the following conditions are equivalent:

$1^{\circ} \mathrm{i} T$ is selfadjoint,

$2^{\circ}\|G(t) h\|^{p}+\|G(-t) h\|^{p} \leqslant 2\|h\|^{p}$ for all $h \in \mathcal{H} \backslash\{0\}$ and for all $t \in \mathbb{R}$,

$3^{\circ}$ the function $\mathbb{R} \ni t \mapsto\|G(t) h\|^{p}$ is concave for every $h \in \mathcal{H} \backslash\{0\}$.

Acknowledgements. The author thanks the referee for his helpful suggestions concerning the questions discussed in Remarks 3.2 and 3.6.

\section{References}

[1] J. Agler, Hypercontractions and subnormality, J. Operator Theory 13 (1985), 203-217.

[2] A. Athavale, On completely hyperexpansive operators, Proc. Amer. Math. Soc. 124 (1996), 3745-3752.

[3] B. Aupetit and J. Zemánek, A characterization of normal matrices by their exponentials, Linear Algebra Appl. 132 (1990), 119-121.

[4] B. Aupetit and J. Zemánek, Erratum: "A characterization of normal matrices by their exponentials" (Linear Algebra Appl. 132 (1990), 119-121), Linear Algebra Appl. 180 (1993), $1-2$.

[5] S. K. Berberian, Some conditions on an operator implying normality, Math. Ann. 184 (1970), 188-192.

[6] J. Daneš, On local spectral radius, Časopis Pěst. Mat. 112 (1987), 177-187.

[7] A. Daniluk and J. Stochel, Seminormal composition operators induced by affine transformations, Hokkaido Math. J. 26 (1997), 377-404.

[8] S. Friedland, A characterization of normal matrices, Israel J. Math. 42 (1982), 235-240.

[9] T. Furuta, On the class of paranormal operators, Proc. Japan Acad. 43 (1967), 594-598.

[10] V. Istrătescu, T. Saitô and T. Yoshino, On a class of operators, Tôhoku Math. J. 18 (1966), 410-413.

[11] Z. J. Jabłoński and J. Stochel, Unbounded 2-hyperexpansive operators, Proc. Edinburgh Math. Soc. 44 (2001), 613-629.

[12] V. Müller, Orbits, weak orbits and local capacity of operators, Integr. Equ. Oper. Theory 41 (2001), 230-253.

[13] S. Ôta, Unbounded nilpotents and idempotents, J. Math. Anal. Appl. 132 (1988), 300-308.

[14] S. Richter, Invariant subspaces of the Dirichlet shift, J. Reine Angew. Math. 386 (1988), 205-220. 
[15] F. Riesz and B. Sz.-Nagy, Leçons d'analyse fonctionnelle, Akadémiai Kiadó, Budapest, 1972.

[16] V. M. Sholapurkar and A. Athavale, Completely and alternatingly hyperexpansive operators, J. Operator Theory 43 (2000), 43-68.

[17] J. Stochel, Seminormal composition operators on $L^{2}$ spaces induced by matrices, Hokkaido Math. J. 19 (1990), 307-324.

[18] P. Vrbová, On local spectral properties of operators in Banach spaces, Czechoslovak Math. J. 23 (1973), 483-492. 